\title{
MAXIMAL SUMS OF ORDINALS
}

\author{
BY \\ BEN DUSHNIK
}

1. Definitions $\left({ }^{1}\right)$. Consider a sum of the type

$$
\sum_{\xi<\beta} a_{\xi}
$$

where $\beta$ is an ordinal, and where $a_{\xi}$ for every $\xi<\beta$ is likewise an ordinal. We shall call $\beta$ the argument of the sum (I). The summands of (I) need not be all distinct; the cardinal number of times that a given ordinal appears as a summand of (I) may be called the multiplicity of that summand. The set of all distinct summands of a sum, each with its multiplicity, may be called the system of summands. We shall use the notation $S \equiv[\alpha|a, \beta| b, \cdots]$ to denote a system $S$ of summands, indicating that the ordinal $\alpha$ is used $a$ times as a summand, where $a$ is a cardinal number, and so on.

By the class-number of an ordinal $\beta$ we shall mean the aleph $\aleph_{\gamma}$ such that the power of an ordered set of type $\beta$ is equal to $\aleph_{\gamma}$ (if $\omega_{\mu}$ is an initial ordinal, its class-number will thus be $\boldsymbol{\aleph}_{\mu}$ ).

A system $S$ of summands will be called appropriate to a given ordinal $\beta$, if the system can be arranged over $\beta$ into a sum of type (I). In order that $S$ be appropriate to $\beta$, it is obviously necessary (and sufficient) that the sum of the multiplicities of the different summands of $S$ be equal to the classnumber of $\beta$.

Let $S$ be a system of summands appropriate to the ordinal $\beta$, and let

$$
\sum_{1} \equiv \sum_{\xi<\beta} a_{\xi}
$$

be an arrangement of $S$ over $\beta$ into a sum of such a nature that if

$$
\sum_{2} \equiv \sum_{\xi<\beta} a_{\xi}^{\prime}
$$

is any other arrangement of $S$ over $\beta$, then

$$
\sum_{2} \leqq \sum_{1}
$$

In such a case we shall say that $\sum_{1}$ is a maximal sum (of $S$ over $\beta$ ).

Let $\beta$ be an ordinal of the form $\beta=\alpha \cdot \gamma+\delta$ where $\alpha$ is an initial ordinal and where $0 \leqq \delta<\alpha$, and let $\xi$ be an ordinal such that $0 \leqq \xi<\gamma$. Then the set

Presented to the Society, September 13, 1935; received by the editors August 7, 1946.

(1) Throughout this paper we assume as known the elementary properties of, and the usual terminology in connection with, ordinal numbers. See, for example, Hausdorff's Grundzuge der Mengenlehre, 1st ed., 1914, especially chap. 5. 
of ordinals $\mu$ such that $\alpha \cdot \xi \leqq \mu<\alpha(\xi+1)$ will be called an $\alpha$-band of the number $\beta$.

We shall say that an $\alpha$-band of an ordinal $\beta$ can be saturated with respect to a system $S$ of summands, if it is possible to choose from $S$ a series of summands

$$
(\alpha): a_{1}, a_{2}, \cdots, a_{\xi}, \cdots \quad(\xi<\alpha)
$$

of type $\alpha$ (where any summand $a_{\xi}$ may be chosen any cardinal number of times not exceeding its multiplicity in $S$ ) such that the sum $\sum_{\xi<\alpha} a_{\xi}$ is as large as any other such sum, obtained by a different choice of the series $(\alpha)$.

By a sum-rest $C_{\gamma}$ of a sum of type (I) we shall understand the sum

$$
C_{\gamma} \equiv \sum_{\gamma \leqq \xi<\beta} a_{\xi}
$$

By an argument strictly analogous to the one used in the case of the rests of any ordinal, it can be readily shown that the set of all different sum-rests of a given sum is finite.

If $\alpha$ is the greatest ordinal such that $\beta^{\alpha} \leqq \gamma$, then $\alpha$ will be called the $e x$ ponent of $\gamma$ with respect to $\beta$, and this will be denoted by writing

$$
\alpha=\exp (\gamma \mid \beta) \text {. }
$$

2. Introduction. Since the addition of ordinals is not commutative, it is clear that, having given a system of summands $S$ and an ordinal $\beta$ to which $S$ is appropriate, it is in general possible to obtain different sums of type (I) by different arrangements of $S$ over $\beta$. It is thus of interest to inquire under what conditions the set of all such different sums contains a maximal sum. This paper is concerned with conditions upon the argument of the sum alone, which insure the existences of the maximal sum no matter what the appropriate system of summands may be. An obvious condition of this nature is that the argument be a finite ordinal. A more interesting condition of this nature is contained in Theorem II of this paper. Theorem I serves then to indicate that the (sufficient) condition of Theorem II is necessary.

3. Some preliminary lemmas. We start with a few simple considerations which will facilitate the proofs of the main theorems to follow in later sections.

LEMMA a. If $\beta$ is an ordinal of the form

$$
\beta=\alpha \cdot \gamma,
$$

where $\alpha$ is an initial ordinal, and if the appropriate system of summands $S$ is such that all the $\alpha$-bands of $\beta$ may be saturated simultaneously (and independently of one another) with respect to $S$, then there exists an arrangement of $S$ over $\beta$ for which the resulting sum is maximal.

For example, suppose $\beta=\Omega \cdot 2$, where $\Omega$ is the smallest transfinite ordinal of the third class of Cantor, and let $S \equiv\left[\Omega\left|1, \Omega^{2}\right| 1, \cdots, \Omega^{\xi} \mid 1, \cdots\right]$, for all 
$\xi<\Omega$. Then the sum

$$
\sum \equiv \sum_{\xi<\Omega} \Omega^{2 \xi}+\sum_{\xi<\Omega} \Omega^{2 \xi+1}=\Omega^{\Omega \cdot 2}
$$

represents a simultaneous saturation of the $2 \Omega$-bands of $\beta$ with respect to $S$, and $\sum$ is indeed the maximal sum of $S$ over $\beta$.

We shall refer to this lemma hereafter as the "saturation principle."

LEMMA b. Let $\sum_{\xi<\beta} a_{\xi}$ be any sum of ordinals $a_{\xi}$ over the well-ordered argument $\beta$, and let $K:\left(k_{1}, k_{2}, \cdots, k_{n}\right)$ be any finite set of ordinals. Then the number of different-valued sums that can be obtained from $\sum_{\xi<\beta} a_{\xi}$ by inserting into it, as additional summands, all the elements of the set $K$ in any order whatever is finite.

Proof. We have already noticed that the set of all different sum-rests of a given sum is finite. It follows readily that there exists a (unique) finite set of indices $0=\xi_{1}<\xi_{2}<\cdots<\xi_{\iota}<\beta(s \geqq 1)$ such that $\xi_{2}$ is the smallest ordinal for which

$$
\sum_{\xi_{2} \leqq \xi<\beta} a_{\xi}<\sum_{\xi<\beta} a_{\xi},
$$

$\xi_{3}$ is the smallest ordinal greater than $\xi_{2}$ for which

$$
\sum_{\xi_{3} \leq \xi<\beta} a_{\xi}<\sum_{\xi_{2} \leqq \xi<\beta} a_{\xi},
$$

and so on; in a word, these ordinals give the finite number of the successive "critical" indices in the argument $\beta$, where the sum-rests first change in value.

Let now $\sum^{\prime}$ be a sum obtained from $\sum_{\xi<\beta} a_{\xi}$ by inserting the ordinals of the set $K$ into it, and let $\bar{k}_{1}, \bar{k}_{2}, \cdots, \bar{k}_{n}$ be any permutation of the $n$ ordinals of this set. Consider the portion of $\sum^{\prime}$ between $a_{\xi_{i}}$ and $a_{\xi_{i+1}}, i<s$ :

$$
\begin{aligned}
\sum^{\prime \prime} \equiv & a_{\xi_{i}}+\cdots+\bar{k}_{j}+\alpha_{v^{\prime}}+\cdots+\bar{k}_{j+1}+a_{v^{\prime \prime}}+\cdots \\
& +\bar{k}_{j+l}+a_{v}+\cdots+a_{\xi_{i+1}}
\end{aligned}
$$

where $\xi_{i}<v^{\prime}<v^{\prime \prime}<\ldots<v<\xi_{i+1}, j+l \leqq n$, and where we insist that no fur ther insertions exist between $a_{v}$ and $a_{\xi_{i+1}}$, except possibly those immediately preceding $a_{\xi_{i+1}}$.

Since the $\xi_{i}, i=1,2, \cdots, s$, are "critical" indices in the sense explained above, we have

$$
\sum_{\xi i \leq \xi<\mu} a_{\xi}=\sum_{\mu \leqq \xi<\beta} a_{\xi}
$$

for $\xi_{i} \leqq \mu<\xi_{i+1}$, so that for any such $\mu$

$$
\exp \left(\sum_{\xi_{i} \leqq \xi<\beta} a_{\xi} \mid \omega\right)<\exp \left(\sum_{\mu \leqq \xi<\beta} a_{\xi} \mid \omega\right),
$$

so that, if $\sum^{\prime \prime \prime}$ is the portion of $\sum^{\prime}$ after $\sum^{\prime \prime}$, 


$$
\begin{aligned}
\sum^{\prime \prime}+\sum^{\prime \prime \prime}= & \bar{k}_{j}+\bar{k}_{j+1}+\cdots+\bar{k}_{i+l}+a_{v}+\cdots+a_{\xi_{i+1}}+\sum^{\prime \prime \prime} \\
= & \bar{k}_{j}+\bar{k}_{j+1}+\cdots+\bar{k}_{j+l}+a_{\xi_{i}}+\cdots+a_{v^{\prime}}+\cdots \\
& +a_{\xi_{i+1}}+\sum^{\prime \prime \prime},
\end{aligned}
$$

because of $(B)$. In other words, the value of $\sum^{\prime}$ is not changed if we take all the ordinals inserted between $a_{\xi_{i}}$ and $a_{v}<a_{\xi_{i+1}}$ and insert them instead, in the order in which they occur originally, just before $a_{\xi_{i}}$. But clearly by means of insertions of this sort, that is, where the insertions occur just before the several critical indices, we can obtain only a finite number of different values for $\sum^{\prime}$; this, however, is precisely the conclusion of our lemma.

LEMMA $\mathrm{c}$. With the hypotheses of Lemma $\mathrm{b}$, there exists an insertion of the ordinals of $K$ into $\sum_{\xi<\beta} a_{\xi}$ which is best (in the sense that the value of the sum resulting from this "best" insertion is at least as great as the value obtained by any other insertion).

This is an immediate consequence of the fact that only a finite number of different values can be obtained by means of all possible insertions of the elements of $K$ into the given sum.

4. A necessary condition. We shall now prove the following theorem:

THEOREM I. If the transfinite ordinal $\beta$ is either a nonlimiting number of Cantor's second class, or any number of any higher class, then there exists a system of summands $S$, appropriate to $\beta$, such that no arrangement of $S$ over $\beta$ gives a maximal sum.

Proof. We discuss the two cases separately.

Case 1. Suppose $\beta=\omega \cdot \alpha+n$, where $n \geqq 1$ is any finite ordinal, and $0<\alpha<\omega_{1}$. Consider the system of summands

$$
S \equiv[1|1,2| 1, \cdots, k \mid 1, \cdots], \quad k<\omega .
$$

$S$ is certainly appropriate to $\beta$. Let

$$
\sum_{1} \equiv \sum_{\xi<\beta} a_{\xi}
$$

be any arrangement of $S$ into a sum over the argument $\beta$. The sum of the summands in $\sum_{1}$ over any $\omega$-band of $\beta$ will be $\omega$, while the sum of the last $n$ summands in $\sum_{1}$ will be some finite ordinal $N \geqq n$. Hence, by the associative law of addition,

$$
\sum_{1}=\omega \cdot \alpha+N
$$

Let now $\sum_{2}$ be any arrangement of $S$ over $\beta$ in which one of the last $n$ summands is the ordinal $N+1$. Then

$$
\sum_{2} \geqq \omega \cdot \alpha+N+1>\sum_{1} .
$$


Since $\sum_{1}$ was an arbitrary arrangement of $S$ over $\beta$, it is clear that no maximal sum can exist in this case.

Case 2. Here we suppose $\beta=\omega_{1} \cdot \alpha+\delta$, where $\alpha \geqq 1$ is an arbitrary ordinal, and $0 \leqq \delta<\omega_{1}$. Let the class-number of $\beta$ be $\boldsymbol{\aleph}_{\mu}$ and consider the system of summands

$$
S \equiv\left[\omega_{\mu+1}\left|\boldsymbol{N}_{0}, 1\right| \boldsymbol{\aleph}_{\mu}\right],
$$

which is clearly appropriate to $\beta$. Let $\sum_{1}$ be any arrangement of $S$ over $\beta$, and suppose that $\gamma$ is the order-type of the successive appearances of the summand $\omega_{\mu+1}$ in this arrangement. Since this summand is of denumerable multiplicity, we must have $\omega \leqq \gamma<\omega_{1}$. Moreover, since the multiplicity of the summand 1 is $\aleph_{\mu}$, any series of this summand in $\sum_{1}$ will be "absorbed" by the summand $\omega_{\mu+1}$ which immediately follows this series. All this makes it obvious that

$$
\sum_{1}=\omega_{\mu+1}^{\gamma}+\xi
$$

where $0 \leqq \xi \leqq \beta$. Consider now an arrangement $\sum_{2}$ of $S$ over $\beta$ in which all the summands $\omega_{\mu+1}$ appear in the very first $\omega_{1}$-band of $\beta$, and in which the ordertype of the successive appearances of this summand is $\eta$, where $\eta>\boldsymbol{\gamma}$. All this is surely possible under our hypothesis, and, as before, we can compute $\sum_{2}$ to be precisely $\omega_{\mu+1}^{\eta}+\beta$. We thus have

$$
\sum_{2}=\omega_{\mu+1}^{\eta}+\beta>\omega_{\mu+1}^{\gamma}+\xi=\sum_{1},
$$

so that no maximal sum is possible in this case either.

The proof of Theorem I is now complete.

5. A sufficient condition. Limiting ordinals of the second class of Cantor, unlike the ordinals contemplated in the theorem of the preceding section, behave like finite ordinals when used as arguments in a sum of ordinals; more explicitly, we can demonstrate the following theorem:

THEOREM II. Let

$$
\beta=\omega \cdot \alpha \quad\left(0 \leqq \alpha \leqq \omega_{1}\right)^{\cdot}
$$

be any limiting ordinal of the second class of Cantor, and let $S$ be any system of summands appropriate to $\beta$. Then there exists an arrangement of $S$ over $\beta$ into a sum which is maximal.

Proof. We arrange the system $S$ into a series of type $\delta$ :

$$
\Gamma:\left\{\bar{\gamma}_{1}, \bar{\gamma}_{2}, \cdots, \bar{\gamma}_{\xi}, \cdots\right\} \quad\left(\xi<\delta<\omega_{1}\right),
$$

where $\xi<\xi^{\prime}$ implies $\bar{\gamma}_{\xi} \leqq \bar{\gamma}_{\xi^{\prime}}$, and wherein any summand of $S$ is repeated either $k$ times or $\omega$ times, according as the multiplicity of that summand is the finite cardinal $k$ or $\boldsymbol{N}_{0}$. For convenience, we distinguish between three possible cases. 
Case I. The type $\delta$ of the series $\Gamma$ is a limiting ordinal. Since $\delta$ is confinal with $\omega$, there exists a sequence $\Gamma:\left\{\gamma_{1}, \gamma_{2}, \cdots, \gamma_{n}, \cdots\right\}(n=1,2, \cdots)$ of nondecreasing ordinals taken from $\bar{\Gamma}$, such that, if $\bar{\gamma}_{\xi}(\xi<\delta)$ is any element of $\bar{\Gamma}$, then there is a finite $n$ for which $\gamma_{n} \geqq \bar{\gamma}_{\xi}$. Without loss of generality we may assume that either

(a) $\exp \left(\gamma_{n} \mid \omega\right)=\zeta$, for any natural $n$,

or

(b) the exponents of the elements of $\Gamma$, with respect to $\omega$, increase with $n$, and this set of exponents, when arranged in order of greatness, has the ordinal $\zeta$ as a limit:

$$
\exp \left(\gamma_{i} \mid \omega\right)<\exp \left(\gamma_{j} \mid \omega\right), \quad \text { for } i<j ;
$$

and

$$
\lim _{n} \exp \left(\gamma_{n} \mid \omega\right)=\zeta
$$

Let now $\sum_{\xi \leq u} a_{\xi}$ be the sum of any sequence of elements taken from the series $\Gamma$. We can find a subsequence of the sequence $\Gamma$, such as

$$
\gamma_{k_{1}}, \gamma_{k_{2}}, \cdots, \gamma_{k_{i}}, \cdots \text {, }
$$

such that $k_{1}<k_{2}<\cdots$ and, for every natural $i, \gamma_{k_{i}} \geqq a_{i}$. We shall thus have

$$
\sum_{\xi<\omega} a_{\xi} \leqq \sum_{\xi<\omega} \gamma_{k_{\xi}} \text {. }
$$

But it is clear that the value of the sum on the right in (A) is fixed, no matter what increasing sequence of integers $k_{1}, k_{2}, \cdots$ is taken; indeed, if we have the situation of (a), then

$$
\sum_{\xi<\omega} \gamma_{k_{\xi}}=\omega^{5+1}
$$

while, if (b) holds,

$$
\sum_{\xi<\omega} \gamma_{k_{\xi}}=\omega^{5}
$$

Hence, if $\sum_{\xi<\beta} a_{\xi}$ is any arrangement of $S$ over $\beta$ in which every $\omega$-band contains an infinite subsequence of summands taken from $\Gamma$, then it is clear that all the $\omega$-bands will be simultaneously saturated with respect to $S$, because of inequality (A). It is clear that such an arrangement is always possible; by the saturation principle, such an arrangement necessarily makes the resulting sum maximal.

(The actual value of $\sum_{k<\beta} a_{\xi}$, if it is maximal, is either $\omega^{5+1} \cdot \alpha$ or $\omega^{5} \cdot \alpha$, according as we have before us situation (a) or (b), respectively.)

Case II. Here we suppose that the type $\delta$ of the series $\Gamma$ is a nonlimiting ordinal, 


$$
\delta=\omega \cdot v+\kappa,
$$

$k$ a finite integer not less than 1 ,

and wherein the "associated" series

$$
\Gamma^{\prime}:\left\{\bar{\gamma}_{\xi}\right\}
$$

is of the sort contemplated in case $I$, (a), while the exponents with respect to $\omega$ of the elements in the finite sequence $\Gamma^{\prime \prime}$ terminating $\bar{\Gamma}$,

$$
\Gamma^{\prime \prime}: \bar{\gamma}_{\omega \cdot v}, \bar{\gamma}_{\omega \cdot v+1}, \cdots, \bar{\gamma}_{\omega \cdot v+k-1},
$$

are all equal to $\zeta$.

Now it is clear, from the nature of the exponents of the elements of $\Gamma^{\prime \prime}$ and by the discussion of case $I$, that any sum (over the argument $\beta$ ) that can be obtained by using $\Gamma$ can be duplicated as to its value by a sum obtained by using $\Gamma^{\prime}$, and conversely. However, by case $I$, we can arrange $\Gamma^{\prime}$ over $\beta$ as an argument into a maximal sum; hence, in this case also, $\Gamma$ (that is to say, the system of summands $S$ can be arranged over $\beta$ into a sum which will be maximal).

(The value of the maximal sum here will again be $\omega^{5+1} \cdot \alpha$.)

Case III. Here again, the type $\delta$ of the series $\Gamma$ is a non-limiting ordinal, but in the associated series $\Gamma^{\prime}$ of case II, the situation of case I, (b) is assumed to hold. Hence the exponents with respect to $\omega$ of the elements of the terminating finite sequence $\Gamma^{\prime \prime}$ are here all greater than or equal to $\zeta$, but otherwise arbitrary.

The "associated" series $\Gamma^{\prime}$ satisfies the conditions of case I, (b), and hence there exists an arrangement of $\Gamma^{\prime}$ over $\beta$ as an argument into a maximal sum; let such a sum be denoted by $\bar{\sum}$. We now make the "best" insertion (see Lemma $\mathrm{c}$ of $\S 3$ ) of the ordinals of $\Gamma^{\prime \prime}$ into this sum $\bar{\sum}$. It is clear that after such a best insertion we have before us an arrangement of $\bar{\Gamma}$, that is, of the system $S$, into a sum $\sum \equiv \sum_{\xi<\beta} a_{\xi}$ over the argument $\beta$. I assert that this sum is a maximal sum.

Indeed, let $\Sigma^{\prime}$ represent any other arrangement of $S$ over $\beta$ into a sum. Rearrange the supposedly maximal sum $\sum$ by transposing in it the summands taken from $\Gamma^{\prime \prime}$ into the same positions that they occupy in the sum $\sum^{\prime}$, but not disturbing the relative positions of the summands from $\Gamma^{\prime}$. If the resulting sum is denoted by $\sum^{\prime \prime}$, we clearly have

$$
\Sigma^{\prime \prime} \leqq \sum
$$

since $\sum^{\prime \prime}$ and $\sum$ are obtainable from $\bar{\sum}$ by two different insertions of the ordinals from $\Gamma^{\prime \prime}$, and $\sum$ is supposed to be a best insertion. We now compare $\Sigma^{\prime}$ and $\sum^{\prime \prime}$, and to this end compare any two corresponding $\omega$-bands: let

$$
a^{\prime} \equiv a_{1}^{\prime}+\cdots+A_{1}+a_{k}^{\prime}+\cdots+A_{2}+a_{\imath}{ }^{\prime}+\cdots+A_{j}+a_{m}^{\prime}+\cdots
$$

be any band of $\sum^{\prime}$, which contains only the elements $A_{1}, A_{2}, \cdots, A_{j}$ from 
$\Gamma^{\prime \prime}$, and let

$$
a \equiv a_{1}+\cdots+A_{1}+a_{k}+\cdots+A_{2}+a_{l}+\cdots+A_{j}+a_{m}+\cdots
$$

be the corresponding band of $\sum^{\prime \prime}$. By hypothesis, the exponent with respect to $\omega$ of any $A_{i}$ is not less than $\zeta$, while the corresponding exponents of any $a_{i}$ or $a_{i}^{\prime}$, or of any finite sum of them, is less than $\zeta$. Hence

$$
a=A_{1}+A_{2}+\cdots+A_{i}+\sum_{\xi<\omega} a_{\xi},
$$

and

$$
a^{\prime} \leqq A_{1}+A_{2}+\cdots+A_{j}+\sum_{\xi<\omega} a_{\xi}^{\prime} .
$$

But $\sum_{\xi<\omega} a_{\xi}^{\prime} \leqq \sum_{\xi<\omega} a_{\xi}$ since the $a_{\xi}$ and $a_{\xi}^{\prime}$ are elements of $\Gamma^{\prime}$ and $\sum_{\xi<\omega} a_{\xi}$ represents a saturation of an $\omega$-band of $\beta$ with respect to $\Gamma^{\prime}$.

Thus, from (D) and (E), $a^{\prime} \leqq a$. If $a^{\prime}$ and $a$ are the sums of corresponding bands of $\sum^{\prime}$ and $\sum^{\prime \prime}$ which do not contain summands of $\Gamma^{\prime \prime}$, then we still have the last inequality. Thus any $\omega$-band of $\sum^{\prime}$ has a sum which is not greater than the sum of the corresponding band from $\sum^{\prime \prime}$, so that

$$
\Sigma^{\prime} \leqq \sum^{\prime \prime} \text {. }
$$

Comparing (C) and (F), we have

$$
\sum^{\prime} \leqq \sum
$$

and thus $\sum$ is maximal.

The proof of Theorem II is now complete, since the three cases considered exhaust all possible cases with regard to the system $S$.

6. A related problem. This paper concerned itself entirely with the finding of conditions upon the argument $\beta$ of a sum of type

$$
\sum_{\xi<\beta} a_{\xi}
$$

which insure the existence of a maximal sum, however the appropriate system of summands may have been chosen.

Another sort of problem, related to the problem of this paper, may be of interest:

Given an ordinal $\beta$, characterize those systems $S$, appropriate to $\beta$, which can be arranged over $\beta$ into a maximal sum.

Except for certain trivial instances, I found it difficult to obtain an answer to this problem by the methods employed in the present paper.

UNIVERSITY OF MichigaN,

ANN ARbor, Mich. 